\title{
Renormalization group for phases with broken discrete symmetry near quantum critical points
}

\author{
P. Jakubczyk, ${ }^{1,2,-⿴ 囗 大}$ P. Strack, ${ }^{1}$ A.A. Katanin, ${ }^{1,3}$ and W. Metzner ${ }^{1}$ \\ ${ }^{1}$ Max-Planck-Institute for Solid State Research, Heisenbergstr. 1, D-70569 Stuttgart, Germany \\ ${ }^{2}$ Institute for Theoretical Physics, Warsaw University, Hoża 69, 00-681 Warsaw, Poland \\ ${ }^{3}$ Institute of Metal Physics, 620219 Ekaterinburg, Russia
}

(Dated: November 18, 2018)

\begin{abstract}
We extend the Hertz-Millis theory of quantum phase transitions in itinerant electron systems to phases with broken discrete symmetry. Using a set of coupled flow equations derived within the functional renormalization group framework, we compute the second order phase transition line $T_{c}(\delta)$, with $\delta$ a non-thermal control parameter, near a quantum critical point. We analyze the interplay and relative importance of quantum and classical fluctuations at different energy scales, and we compare the Ginzburg temperature $T_{G}$ to the transition temperature $T_{c}$, the latter being associated with a non-Gaussian fixed-point.

PACS numbers: 05.10.Cc, 73.43.Nq, 71.27.+a
\end{abstract}

\section{INTRODUCTION}

Quantum phase transitions in itinerant electron systems continue to ignite considerable interest. ${ }^{1.2 .3}$ These transitions are usually induced by varying a non-thermal control parameter such as pressure or chemical potential at zero temperature, so that no classical fluctuations occur and the transition is driven exclusively by quantum effects. In many physical situations, a line of finite temperature second order phase transitions in the phase diagram terminates at a quantum critical point at $T=0$. In such cases quantum fluctuations influence the system also at finite temperatures altering measurable quantities such as the shape of the phase boundary. Consequently, in a complete description of the system at finite $T$, quantum and thermal fluctuations have to be accounted for simultaneously.

The conventional renormalization group (RG) approach to quantum criticality in itinerant electron systems $\frac{4.5}{}$ relies on the assumption that it is sensible to integrate out fermionic degrees of freedom from the functional integral representation of the partition function and then to expand the resulting effective action in powers of the order parameter alone. This approach has been questioned for magnetic phase transitions associated with spontaneous breaking of continuous spin rotation invariance, since integrating the fermions results in singular interactions of the order parameter field, ${ }^{2,3}$

In this paper, we focus on quantum phase transitions to phases with broken discrete symmetry. We analyze quantum and classical fluctuations in the symmetry-broken phase with an Ising-like order parameter near a quantum critical point. Our calculations are based on a set of coupled flow equations obtained by approximating the exact flow equations of the one-particle irreducible version of the functional RG. Quantum and classical (thermal) fluctuations are treated on equal footing. The functional RG has been applied extensively to classical critical phenomena, $\frac{6}{-}$ where it provides a unified description of $O(N)$-symmetric scalar models, including two-dimensional systems. The classical Ising universality class has been analyzed in Refs. 7,8,9. The functional RG is obviously also a suitable framework for treating quantum criticality $\stackrel{10}{=}$ In our approach, we can compute the RG flow in any region of the phase diagram, including the region governed by non-Gaussian critical fluctuations. This allows comparison between the true transition line and the Ginzburg line, which so far has been used as an estimate of the former ${ }^{3.5}$ Specifically, we capture the strong-coupling behavior emergent in the vicinity of the transition line as well as the correct classical fixed-point for $T_{c}$, including the anomalous dimension of the order parameter field.

Our results may be applied to commensurate chargedensity-waves, Pomeranchuk transitions ${ }^{11,12,13}$ which spontaneously break the discrete point group lattice symmetry, and to magnetic transitions with Ising symmetry, provided the transitions are continuous at low temperatures.

The paper is structured as follows: in Section II we introduce Hertz's 4 effective action, adapted to the symmetrybroken phase, which serves as a starting point for the subsequent analysis. In Section III we describe the functional RG method and its application in the present context, and subsequently derive the RG flow equations. In Section IV we present a solution of the equations in the case $T=0$. Section $\mathrm{V}$ contains numerical results for the finite $T$ phase diagram in the region with broken symmetry. Different cases are discussed, distinguished by the dimensionality $d$ and the dynamical exponent $z$. In particular, we compare the $T_{c}$ line with the Ginzburg line, thus providing an estimate of the critical region size. In Section VI we summarize and discuss the results.

\section{HERTZ ACTION}

The starting point of the standard RG approach to quantum critical phenomena in itinerant electron systems is the Hertz action ${ }^{4,5}$ It can be derived from a microscopic Hamiltonian by applying a Hubbard-Stratonovich transformation to the pathintegral representation of the partition function and subsequently integrating out the fermionic degrees of freedom. The resulting action is then expanded in powers of the order parameter field, usually to quartic order. The validity of this expansion is dubious in several physically interesting cases, in particular for magnetic transitions with $\mathrm{SU}(2)$-symmetry, since the integration over gapless fermionic modes can lead to singular effective interactions of the order parameter field, 
which may invalidate the conventional power counting, $2,3,14$ Such complications probably do not affect transitions in the charge channel and magnetic transitions with Ising symmetry. There are several indications that singularities cancel in that case, namely the cancellation of singularities in effective interactions upon symmetrization of fermion loops,,$\frac{15,16}{17}$ and the cancellation of non-analyticities in susceptibilities $\stackrel{17,18}{=} \mathrm{We}$ therefore rely on the usual expansion of the action to quartic order in the order parameter field and also on the conventional parametrization of momentum and frequency dependences, which leads to the Hertz action ${ }^{4.5}$

$$
S[\phi]=\frac{T}{2} \sum_{\omega_{n}} \int \frac{d^{d} p}{(2 \pi)^{d}} \phi_{p}\left(\frac{\left|\omega_{n}\right|}{\mid \mathbf{p}^{2-2}}+\mathbf{p}^{2}\right) \phi_{-p}+U[\phi] .
$$

Here $\phi$ is the scalar order parameter field and $\phi_{p}$ with $p=$ $\left(\mathbf{p}, \omega_{n}\right)$ its momentum representation; $\omega_{n}=2 \pi n T$ with integer $n$ denotes the (bosonic) Matsubara frequencies. Momentum and energy units are chosen such that the prefactors in front of $\frac{\left|\omega_{n}\right|}{|\mathbf{p}|^{2-2}}$ and $\mathbf{p}^{2}$ are equal to unity. The action is regularized in the ultraviolet by restricting momenta to $|\mathbf{p}| \leq \Lambda_{0}$. The value of the dynamical exponent is restricted to $z \geq 2$. The cases $z=3$, applicable to Pomeranchuk instabilities and Ising ferromagnetism, and $z=2$, relevant for commensurate charge density waves and Ising antiferromagnetism, are of our main interest. Formally, the results of the paper may be applied to systems with arbitrary $z \geq 2$. A comprehensive discussion of the origin of the $\omega_{n}$ dependence of the action is given by Millis $\frac{5}{-}$ (see also Ref.19). Eq. (1) is valid in the limit $\frac{\left|\omega_{n}\right|}{||^{\mid-2}} \ll 1$ which is relevant here since the dominant fluctuations occur only in this regime.

In the symmetric phase the potential $U[\phi]$ is minimal at $\phi=0$, and is usually parametrized by a positive quadratic and a positive quartic term. .5 Since we approach the quantum critical point from the symmetry-broken region of the phase diagram, we assume a potential $U[\phi]$ with a minimum at a non-zero order parameter $\phi_{0}$ :

$$
\begin{aligned}
U[\phi] & =\frac{u}{4 !} \int_{0}^{1 / T} d \tau \int d^{d} x\left(\phi^{2}-\phi_{0}^{2}\right)^{2} \\
& =\int_{0}^{1 / T} d \tau \int d^{d} x\left[u \frac{\phi^{\prime 4}}{4 !}+\sqrt{3 u \delta} \frac{\phi^{3}}{3 !}+\delta \frac{\phi^{\prime 2}}{2 !}\right],
\end{aligned}
$$

where $\phi$ and $\phi^{\prime}$ are functions of $x$ and $\tau$ with $\phi=\phi_{0}+\phi^{\prime}$. The parameter $\delta=u \phi_{0}^{2} / 3$ controls the distance from criticality. Approaching the phase boundary in the $(\delta, T)$-plane from the symmetry-broken phase gives rise to the three-point vertex $\sqrt{3 u \delta}$, which generates an anomalous dimension of the order parameter field already at one-loop level.

Although formally correct as a result of integrating out the fermions, the Hertz action (1) is not a good starting point for symmetry-broken phases with a fermionic gap, such as charge density wave phases or antiferromagnets. A fermionic gap leads to a suppression of the dynamical term (linear in frequency) in the action, since it suppresses low energy particlehole excitations. If not treated by a suitable resummation in the beginning, this effect is hidden in high orders of perturbation theory. $\frac{20}{-}$ We do not deal with this complication in the present paper. However, our results for the transition temperature should not be affected by a gap in the symmetry-broken phase, since it vanishes continuously at $T_{c}$.

\section{METHOD}

To analyze the quantum field theory defined by $S[\phi]$ we compute the flow of the effective action $\Gamma^{\Lambda}[\phi]$ with approximate flow equations, which are derived from an exact functional RG flow equation $, 621,22,23,24$. The effective action $\Gamma^{\Lambda}[\phi]$ is the generating functional for one-particle irreducible vertex functions in presence of an infrared cutoff $\Lambda$. The latter is implemented by adding a regulator term of the form $\int \frac{1}{2} \phi R^{\Lambda} \phi$ to the bare action. The effective action interpolates smoothly between the bare action $S[\phi]$ for large $\Lambda$ and the full effective action $\Gamma[\phi]$ in the limit $\Lambda \rightarrow 0$ (cutoff removed). Its flow is given by the exact functional equation ${ }^{21}$

$$
\frac{d}{d \Lambda} \Gamma^{\Lambda}[\phi]=\frac{1}{2} \operatorname{Tr} \frac{\dot{R}^{\Lambda}}{\Gamma^{(2)}[\phi]+R^{\Lambda}},
$$

where $\dot{R}^{\Lambda}=\partial_{\Lambda} R^{\Lambda}$, and $\Gamma^{(2)}[\phi]=\delta^{2} \Gamma^{\Lambda}[\phi] / \delta \phi^{2}$. In momentum representation $\left(\phi_{p}\right)$, the trace sums over momenta and frequencies: $\operatorname{Tr}=T \sum_{\omega_{n}} \int \frac{d^{d} p}{(2 \pi)^{d}}$. For the regulator function $R^{\Lambda}(\mathbf{p})$ we choose the optimized Litim cutoff ${ }^{25}$

$$
R^{\Lambda}(\mathbf{p})=Z\left(\Lambda^{2}-\mathbf{p}^{2}\right) \theta\left(\Lambda^{2}-\mathbf{p}^{2}\right),
$$

where $Z$ is a renormalization factor (see below).

We approximate $\Gamma^{\Lambda}[\phi]$ by a simplified ansatz with a local potential of the form Eq. (2) characterized by cutoff dependent parameters $u$ and $\phi_{0}$ (or, alternatively, $\delta$ ), and a simple renormalization of the momentum and frequency dependences of the quadratic part of the action via $Z$-factors, leading to an inverse propagator of the form

$$
G^{-1}\left(\mathbf{p}, \omega_{n}\right)=\Gamma^{(2)}\left[\phi=\phi_{0}\right]=Z_{\omega} \frac{\left|\omega_{n}\right|}{|\mathbf{p}|^{z-2}}+Z \mathbf{p}^{2}+\delta+R^{\Lambda}(\mathbf{p}),
$$

where the $Z$-factors depend on the scale $\Lambda$ only. The regulator function $R^{\Lambda}(\mathbf{p})$ replaces $Z \mathbf{p}^{2}$ with $Z \Lambda^{2}$ for $|\mathbf{p}|<\Lambda$.

Evaluating Eq. (3) for a momentum-independent field $\phi$ yields the flow of the effective potential $U[\phi]$

$$
\partial_{\Lambda} U[\phi]=\frac{1}{2} \operatorname{Tr} \frac{\dot{R}^{\Lambda}(\mathbf{p})}{Z_{\omega} \frac{\left|\omega_{n}\right|}{\mathbf{p}^{z-2}}+Z \mathbf{p}^{2}+R^{\Lambda}(\mathbf{p})+U^{\prime \prime}[\phi]},
$$

from which we derive the flows of the parameters $\phi_{0}$ and $u$, following the procedure in Ref. 6. Viewing $U$ as a function of $\rho=\frac{1}{2} \phi^{2}$ and using $U^{\prime}\left[\rho_{0}\right]=0$, we can write $0=\frac{d}{d \Lambda} U^{\prime}\left[\rho_{0}\right]=$ $\partial_{\Lambda} U^{\prime}\left[\rho_{0}\right]+U^{\prime \prime}\left[\rho_{0}\right] \partial_{\Lambda} \rho_{0}$. Inserting $\partial_{\Lambda} U^{\prime}\left[\rho_{0}\right]$ as obtained by differentiating Eq. (6) with respect to $\rho$ at $\rho=\rho_{0}$, and using $U^{\prime \prime}\left[\rho_{0}\right]=\frac{1}{3} u$, one obtains the flow equation for $\rho_{0}$

$$
\partial_{\Lambda} \rho_{0}=\frac{3}{2} \operatorname{Tr} \frac{\dot{R}^{\Lambda}(\mathbf{p})}{\left[Z_{\omega} \frac{\left|\omega_{n}\right|}{\mathbf{p}^{z-2}}+Z \mathbf{p}^{2}+R^{\Lambda}(\mathbf{p})+\frac{2}{3} u \rho_{0}\right]^{2}} .
$$


The flow of $u$ is obtained by differentiating Eq. (6) twice with respect to $\rho$ :

$$
\partial_{\Lambda} u=3 u^{2} \operatorname{Tr} \frac{\dot{R}^{\Lambda}(\mathbf{p})}{\left[Z_{\omega} \frac{\left|\omega_{n}\right|}{\mathbf{p}^{z-2}}+Z \mathbf{p}^{2}+R^{\Lambda}(\mathbf{p})+\frac{2}{3} u \rho_{0}\right]^{3}} .
$$

Inserting the above flow equations into the $\Lambda$-derivative of $\delta=$ $\frac{2}{3} u \rho_{0}$, we obtain the flow of $\delta$. Below we will analyze the flow in terms of $u$ and $\delta$ instead of $u$ and $\rho_{0}$.

The Matsubara frequency sums in the flow equations (7) and (8) can be expressed in terms of polygamma functions $\Psi_{n}(z)$, defined recursively by $\Psi_{n+1}(z)=\Psi_{n}^{\prime}(z)$ for $n=$ $0,1,2, \ldots$, and $\Psi_{0}(z)=\Gamma^{\prime}(z) / \Gamma(z)$, where $\Gamma(z)$ is the gamma function. From the Weierstrass representation of the gamma function, $\Gamma(z)^{-1}=z e^{\gamma z} \prod_{n=1}^{\infty}\left(1+\frac{z}{n}\right) e^{-z / n}$, where $\gamma$ is the Euler constant, one can derive the relation

$$
\sum_{n=-\infty}^{\infty} \frac{1}{(|n|+z)^{2}}=\frac{1}{z^{2}}+2 \Psi_{1}(z+1)
$$

Taking derivatives with respect to $z$ yields expressions for sums involving higher negative powers of $(|n|+z)$ in terms of polygamma functions of higher order.

For $R^{\Lambda}(\mathbf{p})$ we now insert the Litim function defined in Eq. (4). Following Ref. 6 we neglect $\dot{Z}$ in $\dot{R}^{\Lambda}(\mathbf{p})$, such that $\dot{R}^{\Lambda}(\mathbf{p})=2 Z \Lambda \Theta\left(\Lambda^{2}-\mathbf{p}^{2}\right)$. The neglected terms would yield corrections to the flow equations that are linear in $\eta$ and therefore irrelevant in the quantum part of the flow, where $\eta \approx 0$ (see below). In the classical part of the flow (for $\Lambda \rightarrow 0$ ) these corrections are small as compared to other included terms involving $\eta$ due to the presence of additional factors involving the interaction coupling and mass. Therefore they give rise to only minor corrections to the values of the fixed point coordinates and the values of the critical exponents.

The $d$-dimensional momentum integrals on the right hand side of the flow equations can be reduced to one-dimensional integrals, since the integrands depend only on the modulus of p.

Explicit dependences on $\Lambda, Z$-factors, and lengthy numerical prefactors in the flow equations can be eliminated by using the following rescaled variables:

$$
\begin{aligned}
\tilde{p} & =|\mathbf{p}| / \Lambda, \\
\tilde{T} & =\frac{2 \pi Z_{\omega}}{Z \Lambda^{z}} T, \\
\tilde{\delta} & =\frac{\delta}{Z \Lambda^{2}}, \\
\tilde{u} & =\frac{A_{d} T}{2 Z^{2} \Lambda^{4-d}} u,
\end{aligned}
$$

with $A_{d}=(2 \pi)^{-d} S_{d-1}$, where $S_{d-1}=2 \pi^{d / 2} / \Gamma(d / 2)$ is the area of the $(d-1)$-dimensional unit sphere.

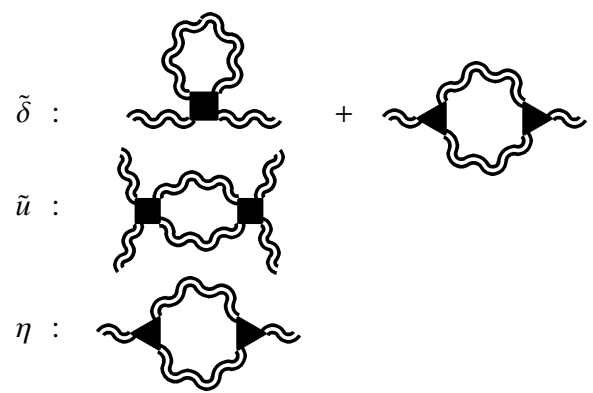

FIG. 1: Feynman diagrams representing the contributions to the flow equations 14[15:19].

The flow equations for $\tilde{\delta}$ and $\tilde{u}$ are then obtained as

$$
\begin{aligned}
\frac{d \tilde{\delta}}{d \log \Lambda} & =(\eta-2) \tilde{\delta} \\
& +4 \tilde{u}\left[\frac{1}{d} \frac{1}{(1+\tilde{\delta})^{2}}+\frac{2}{\tilde{T}^{2}} \int_{0}^{1} d \tilde{p} \tilde{p}^{d+2 z-5} \Psi_{1}(x)\right] \\
& +12 \tilde{u} \tilde{\delta}\left[\frac{1}{d} \frac{1}{(1+\tilde{\delta})^{3}}-\frac{1}{\tilde{T}^{3}} \int_{0}^{1} d \tilde{p} \tilde{p}^{d+3 z-7} \Psi_{2}(x)\right] \\
\frac{d \tilde{u}}{d \log \Lambda} & =(d-4+2 \eta) \tilde{u} \\
& +12 \tilde{u}^{2}\left[\frac{1}{d} \frac{1}{(1+\tilde{\delta})^{3}}-\frac{1}{\tilde{T}^{3}} \int_{0}^{1} d \tilde{p} \tilde{p}^{d+3 z-7} \Psi_{2}(x)\right],
\end{aligned}
$$

where $x=1+\tilde{T}^{-1}(1+\tilde{\delta}) \tilde{p}^{z-2}$, and $\eta$ is the anomalous dimension defined as

$$
\eta=-\frac{d \log Z}{d \log \Lambda}
$$

To complete the system of flow equations one still needs to derive the evolution of $Z$ and $Z_{\omega}$, which parametrize the momentum and frequency dependence of the propagator. Taking the second functional derivative of Eq. (3), we obtain the flow equation for the propagator

$$
\partial_{\Lambda} G^{-1}(p)=3 u \delta \operatorname{Tr}\left[\dot{R}^{\Lambda}(\mathbf{q}) G^{2}(q) G(q+p)\right],
$$

where $G(q)$ is given by Eq. (5). Here we skipped the contribution from the tadpole diagram, since it involves no dependence on momentum and frequency, and therefore does not contribute to the flow of the $Z$-factors.

The momentum renormalization factor is given by

$$
Z=\left.\frac{1}{2 d} \Delta_{\mathbf{p}}\left[G^{-1}\left(\mathbf{p}, \omega_{n}=0\right)\right]\right|_{\mathbf{p}=0},
$$

where $\Delta_{\mathbf{p}}$ is the Laplace operator evaluated at constant cutoff function, that is, $\Delta_{\mathbf{p}}$ does not act on $R^{\Lambda}(\mathbf{p})$. Inserting Eqs. (17) and (18) into Eq. (16), and performing the frequency sum, one 
obtains the flow equation for the anomalous dimension

$$
\begin{aligned}
\eta= & \frac{6}{d} \frac{\tilde{u} \tilde{\delta}}{(1+\tilde{\delta})^{5}}\left[2(1+\tilde{\delta})-\frac{8}{d+2}\right]-\frac{\tilde{u} \tilde{\delta}}{d} \tilde{T}^{-5} \int_{0}^{1} d \tilde{p} \tilde{p}^{d+3 z-13} \\
& {\left[-6 \tilde{p}^{4}(z-2)(d+z-4) \tilde{T}^{2} \Psi_{2}(x)-2 \tilde{p}^{2+z}(2(8-d)\right.} \\
& \left.\left(1+\tilde{\delta}-\tilde{p}^{2}\right)+\left[(d-14)(1+\tilde{\delta})+8 \tilde{p}^{2}\right] z+3(1+\tilde{\delta}) z^{2}\right) \\
& \left.\tilde{T} \Psi_{3}(x)-\tilde{p}^{2 z}\left[2\left(\tilde{p}^{2}-1\right)+\tilde{\delta}(z-2)+z\right]^{2} \Psi_{4}(x)\right]
\end{aligned}
$$

The contributions to the flow of $\tilde{\delta}, \tilde{u}$ and $\eta$ are illustrated in terms of Feynman diagrams in Fig. 1.

We now turn to the renormalization of $Z_{\omega}$. We first consider the case $z=2$, where $Z_{\omega}$ can be related to the propagator by

$$
Z_{\omega}=\frac{1}{2 \pi T}\left[G^{-1}(\mathbf{p}=0,2 \pi T)-G^{-1}(\mathbf{p}=0,0)\right] .
$$

Taking the logarithmic derivative with respect to $\Lambda$, inserting Eq. (17), and performing the trace yields

$$
\eta_{\omega}=\frac{12}{d} \tilde{u} \tilde{\delta} \tilde{T}^{-1}\left[\frac{\tilde{T}-(1+\tilde{\delta})}{\tilde{T}(1+\tilde{\delta})^{3}}+\tilde{T}^{-3} \Psi_{2}\left(1+\frac{1+\tilde{\delta}}{\tilde{T}}\right)\right] .
$$

From the numerical solution of the flow equation we observe that $\eta_{\omega}$ is small at all scales and vanishes for $\Lambda \rightarrow 0$. For example, in two dimensions $\eta_{\omega}$ varies between -0.033 and 0.005 for $u=1$ at $T=e^{-4}$ and has practically no influence on the phase diagram. In three dimensions the values are at least one order of magnitude lower. For $z>2$ one expects an even smaller $\eta_{\omega}$, since a larger $z$ reduces the strength of fluctuations near the quantum critical point. Therefore we set $\eta_{\omega}=0$ and $Z_{\omega}=1$ from now on. The scaling variable $\tilde{T}$ then obeys the flow equation

$$
\frac{d \tilde{T}}{d \log \Lambda}=(\eta-z) \tilde{T}
$$

In the flow equations (14[15]19) one identifies the classical mean-field (involving only one power of $\tilde{\delta}$ or $\tilde{u}$ ), classical non-gaussian and quantum (involving $\tilde{T}$ ) terms. The quantum contributions vanish as the infrared cutoff tends to zero at constant non-zero temperature $\left(\tilde{T}^{-1} \ll 1\right)$. On the other hand the quantum terms dominate the high energy part of the flow, where $\tilde{T}^{-1} \gg 1$. Our framework allows for a continuous connection of these two regimes of the flow. The scale $\Lambda_{c l}$ below which quantum fluctuations become irrelevant depends on temperature. It vanishes at $T=0$. In the absence of a sizable anomalous dimension $\eta$ in the quantum regime of the flow, one has

$$
\Lambda_{c l} \propto T^{1 / z}
$$

as follows directly from the definition of $\tilde{T}$. It turns out that $\eta$ is indeed negligible down to the scale $\Lambda_{c l}$, except in the case $z=2$ in two dimensions (see Sec. V]).

We emphasize that we have used a relatively simple parametrization of the effective action $\Gamma^{\Lambda}[\phi]$. In particular, we kept only the dominant term in the derivative expansion ${ }^{6}$ and neglected the field dependence of the $Z$-factors. Furthermore, the simple parametrization of the effective potential Eq. (2) allowed us to substitute the partial differential equation (6) governing the flow of $U[\phi]$ by the two ordinary differential equations (14) and (15). The latter approximation is equivalent to neglecting all higher order vertices generated during the flow. More sophisticated truncations have been applied in the context of the classical Ising universality class, where they lead to improved results for the critical exponents. ${ }^{7,8}$

\section{SOLUTION AT ZERO TEMPERATURE}

In this section we present a solution of the flow equations 14 15 19] in the case of zero temperature, recovering earlier results for the critical exponents. We also provide an analytic expression for the value of the control parameter $\delta_{0}$ corresponding to the quantum critical point.

For this purpose we first take advantage of the asymptotic properties of the polygamma functions $\Psi_{1}(x)=x^{-1}+O\left(x^{-2}\right)$ for $x \gg 1, \Psi_{n+1}(x)=\Psi_{n}^{\prime}(x)$, and evaluate the integrals in Eqs. 14[15]19] in the regime $\tilde{T} \ll 1$. We introduce the variable

$$
\tilde{v}=\frac{\tilde{u}}{\tilde{T}}=\frac{A_{d}}{4 \pi Z_{\mathbf{p}} Z_{\omega} \Lambda^{4-d-z}} u
$$

and then pass to the limit $T \rightarrow 0$. Note that $\tilde{v}$ does not depend on temperature. The resulting flow equations are

$$
\begin{aligned}
\frac{d \tilde{\delta}}{d \log \Lambda} & =(\eta-2) \tilde{\delta}+\frac{12 \tilde{\delta} \tilde{v}}{(1+\tilde{\delta})^{2}} \frac{1}{d+z-2}+\frac{8 \tilde{v}}{1+\tilde{\delta}} \frac{1}{d+z-2} \\
\frac{d \tilde{v}}{d \log \Lambda} & =(d+z-4+2 \eta) \tilde{v}+\frac{12 \tilde{v}^{2}}{(1+\tilde{\delta})^{2}} \frac{1}{d+z-2} \\
\eta & =\frac{6}{d} \frac{\tilde{\delta} \tilde{v}}{(1+\tilde{\delta})^{4}}\left[\frac{4}{3}(1+\tilde{\delta})\right. \\
& \left.-\frac{(d-2)(z-2)}{3(d+z-4)}(1+\tilde{\delta})^{2}-\frac{4}{d+z}\right] .
\end{aligned}
$$

The above expression for $\eta$ is valid for $d+z>4$. The flow equations for the case $d=z=2$ are obtained by skipping the term involving $(d-2)(z-2) /(d+z-4)$ in Eq. (25). Non-gaussian contributions to the above equations originate from the quantum part of Eqs. (14:15 19). The classical nongaussian terms do not survive the limit $T \rightarrow 0$.

Eqs. (25) have an infrared stable Gaussian fixed point in $\tilde{v}=0, \tilde{\delta}=0, \eta=0$. Linearizing the flow equations around the fixed point, one obtains the solution

$$
\begin{aligned}
\tilde{\delta}(\Lambda) & =\left[\tilde{\delta}^{*}+\frac{8 \tilde{v}^{*}}{(d+z-2)^{2}}\left(\left(\Lambda / \Lambda_{0}\right)^{d+z-2}-1\right)\right]\left(\Lambda / \Lambda_{0}\right)^{-2} \\
\tilde{v}(\Lambda) & =\left(\Lambda / \Lambda_{0}\right)^{d+z-4} \tilde{v}^{*} \\
\eta(\Lambda) & =0
\end{aligned}
$$

for $d+z>4$. Here $\tilde{\delta}^{*}$ and $\tilde{v}^{*}$ on the right hand sides denote the initial values of the parameters at $\Lambda=\Lambda_{0}$. In the marginal 
case $d+z=4$ one finds logarithmic convergence of $\tilde{v}(\Lambda)$ and $\tilde{\delta}(\Lambda)$ to zero. Expressing the order parameter $\phi_{0}$ in terms of $\tilde{\delta}^{*}$ and $\tilde{v}^{*}$, substituting the above solution, and taking the limit $\Lambda \rightarrow 0$ yields

$$
\phi_{0} \propto \sqrt{\delta-\delta_{0}}
$$

where

$$
\delta_{0}=\frac{2 A_{d}}{\pi} \frac{\Lambda_{0}^{d+z-2}}{(d+z-2)^{2}} u
$$

is the quantum critical point's coordinate. From Eq. 27) we read off the value of the exponent $\beta=\frac{1}{2}$, consistent with mean-field theory. From Eq. (26) we can also straightforwardly evaluate the correlation length $\xi$, using $\xi^{-2}=$ $\lim _{\Lambda \rightarrow 0} \delta(\Lambda)=\lim _{\Lambda \rightarrow 0} Z \Lambda^{2} \tilde{\delta}$, which yields

$$
\xi=\left(\delta-\delta_{0}\right)^{-1 / 2},
$$

as expected within mean field theory.

As anticipated, $\stackrel{4,5}{5}$ the quantum phase transitions studied here behave similarly to classical transitions with effective dimensionality $\mathcal{D}=d+z$. For $d \geq 2$ and $z \geq 2$ one has $\mathcal{D} \geq 4$, leading to mean-field behavior governed by a Gaussian fixed point. ${ }^{4}$ In the next section we turn to finite temperatures, where non-Gaussian behavior occurs sufficiently close to the phase transition line.

\section{FINITE TEMPERATURES}

In this section we numerically solve the RG flow equations (141519) for finite temperatures. As already announced, the analysis is focused on the region of the phase diagram where symmetry-breaking occurs. In our notation this corresponds to sufficiently large values of the control parameter $\delta$. First we treat the case $z=3$ (in $d=2,3$ ) to which Eqs. (14 15 19) are applied directly. The case $z=2$, in which additional simplifications occur, is analyzed in Subsection B. In all numerical results we choose an initial cutoff $\Lambda_{0}=1$, and an initial coupling constant $u=1$.

\section{A. $\mathbf{z}=\mathbf{3}$}

We first solve the coupled flow equations (14[15]19) with the aim of determining the phase boundary $T_{c}(\delta)$, or, equivalently $\delta_{c}(T)$. To this end, for each given temperature we tune the initial value of $\delta$ such that at the end of the flow (for $\Lambda \rightarrow 0$ ) one obtains the critical state with $\delta(\Lambda) \rightarrow 0$. The tuned initial value is then identified as $\delta_{c}(T)$. Inverting the function $\delta_{c}(T)$ yields $T_{c}(\delta)$. In the variables $\tilde{\delta}, \tilde{u}$ this corresponds to seeking for such values of the initial $\delta$, that both $\tilde{\delta}(\Lambda)$ and $\tilde{u}(\Lambda)$ reach a fixed point as the cutoff is removed.

The flow of $\eta$ and $\tilde{u}$ as a function of the logarithmic scale variable $s=-\log \left(\Lambda / \Lambda_{0}\right)$ is shown in two exemplary plots in Figs. 2] and 3, respectively. The flow is shown for various temperatures $T$, with $\delta$ tuned to values very close to $\delta_{c}(T)$.

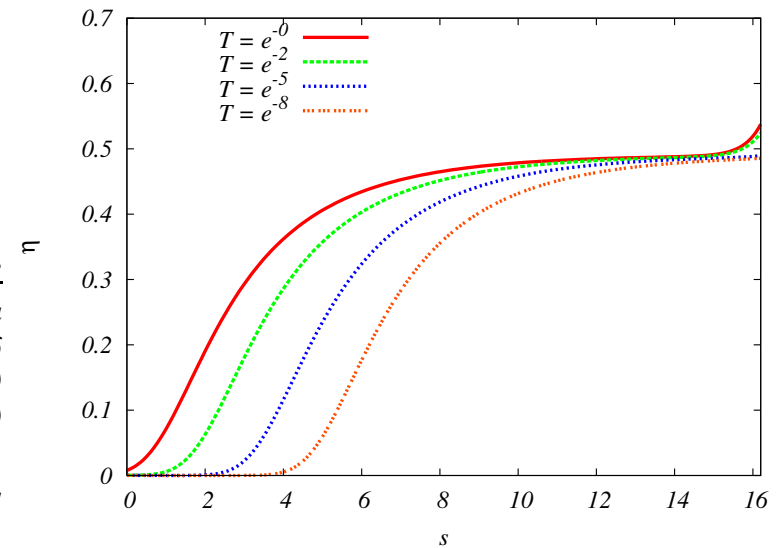

FIG. 2: (Color online) Anomalous dimension $\eta(\Lambda)$ plotted as function of $s=-\log \left(\Lambda / \Lambda_{0}\right)$ for different values of temperature in the case $z=3$ and $d=2$. The function $\eta(s)$ exhibits crossover from the mean-field value $\eta=0$, to the non-gaussian result $\eta \approx 0.48$. The crossover scale $\Lambda_{G}$ is shifted towards smaller values of $\Lambda$ (larger $s$ ) as temperature is reduced.

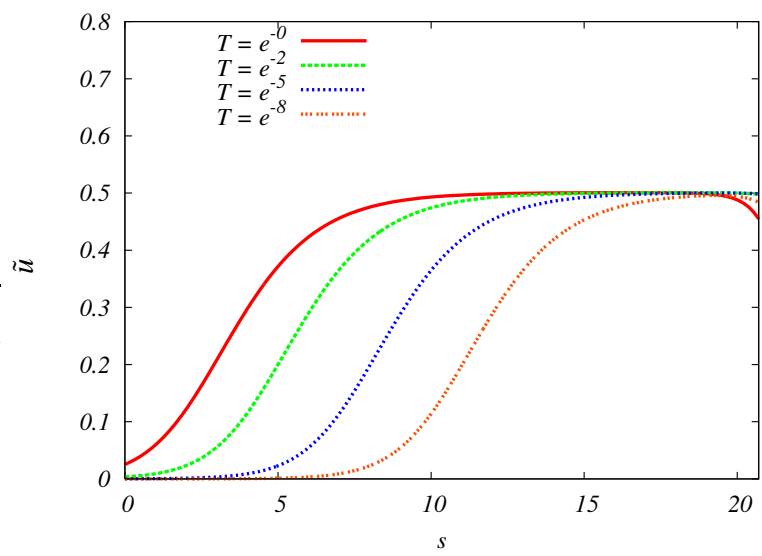

FIG. 3: (Color online) Quartic coupling $\tilde{u}(\Lambda)$ plotted as function of $s=-\log \left(\Lambda / \Lambda_{0}\right)$ for different values of temperature in the case $z=3$ and $d=3$.

The plateaus in Figs. 2 and 3 correspond to non-gaussian fixed point values of the flowing parameters. These values are not altered by the quantum terms in the flow equations. From Fig. 2], where $d=2$, one reads off the value of the anomalous dimension $\eta \approx 0.48$. The exact value from the Onsager solution to the Ising model is $\frac{1}{4}$. For the case $d=3$ we find $\eta \approx 0.08$ within our truncation, which is also about twice as large as accurate estimates of the exact value. ${ }^{26}$ These results can also be compared to earlier classical $\phi^{4}$ RG studies using similar though more sophisticated truncations. In Ref. 7 a derivative expansion to order $\partial^{2}$, retaining the general form of $U[\phi]$ and field dependence of wave function renormalization, was applied in dimensionality $1<d<4$ yielding $\eta \approx 0.08$ in $d=3$ and $\eta \approx 0.4$ in $d=2$. In Ref. 8 a derivative expansion to order $\partial^{4}$ yielded $\eta \approx 0.033$ in $d=3$. To obtain scaling be- 
havior in the range of a few orders of magnitude one needs to fine-tune the initial conditions with an accuracy of around 15 digits. The breakdown of scaling behavior observed for very small $\Lambda$ is due to insufficient accuracy of the initial value of $\delta$ and numerical errors. The plateaus are more extended as we go on fine-tuning the initial condition. Only exactly at the critical point true scale invariance manifested by plateaus of infinite size is expected.

The figures also reveal the Ginzburg scale $\Lambda_{G}$ at which nongaussian fluctuations become dominant, such that the exponent $\eta$ attains a non-zero value. By fitting a power-law we observe $\Lambda_{G} \propto T_{c}$ for $d=3$ and $\Lambda_{G} \propto \sqrt{T_{c}}$ for $d=2$ with nonuniversal proportionality factors. As expected, $\Lambda_{G}$ vanishes at the quantum critical point, because at $T=0$ the effective dimensionality $\mathcal{D}=d+z$ is above the upper critical dimension $d_{c}=4$. At finite temperatures, $\Lambda_{G}$ is the scale at which $\tilde{u}$ is promoted from initially small values (of order $T$ ) to values of order one. From the linearized flow equations one obtains

$$
\Lambda_{G} \propto T_{c}^{\frac{1}{4-d}}
$$

in agreement with the numerical results for $d=2$ and $d=$ 3. Note that $\Lambda_{G} \ll \Lambda_{c l}$, since $\Lambda_{c l} \propto T_{c}^{1 / 3}$ for $z=3$, see Eq. (23). Hence anomalous scaling (finite $\eta$ ) is indeed absent in the regime where quantum fluctuations contribute, and nonGaussian fluctuations appear only in the classical regime.

As already mentioned, the quantum contributions influence the flow only at relatively large $\Lambda$ for $T>0$. In particular, they do not alter any fixed-point values. However, at the beginning of the flow they dominate over the classical part and therefore are crucial for a correct computation of the initial value of $\delta$ leading to a scaling solution in the infrared limit.

Results for the transition line $T_{c}(\delta)$ for $d=2$ and $d=3$ are shown in Fig. 4 . In both cases we recover the shape of the transition line as derived by Millis,,$\frac{5}{-}$ who used the Ginzburg temperature in the symmetric phase as an estimate for $T_{c}$. Namely, we find

$$
\left(\delta-\delta_{0}\right) \propto T_{c} \log T_{c}
$$

for $d=2,27$ and

$$
T_{c} \propto\left(\delta-\delta_{0}\right)^{3 / 4}
$$

for $d=3$. The exponent $3 / 4$ in the three-dimensional case matches with the general formula for the shift exponent, $\psi=\frac{z}{d+z-2}$ for arbitrary $z$ in dimensions $d>2 . \underline{5}$ Note that the phase boundary $T_{c}(\delta)$ approaches the quantum critical point with vanishing first derivative for $d=2$ and with singular first derivative in the case $d=3$. In Ref. 28 functional RG methods in a different truncation were applied to calculate the shift exponent in the context of chiral symmetry breaking with the control parameter being the number of fermion flavours.

An advantage of the present approach is that one can also follow the RG flow into the strong coupling regime, where non-gaussian critical behavior occurs. This in turn allows an estimate of the critical region's size as a function of temperature or the control parameter $\delta$. To evaluate the Ginzburg line in the symmetry-broken phase one solves the flow equations
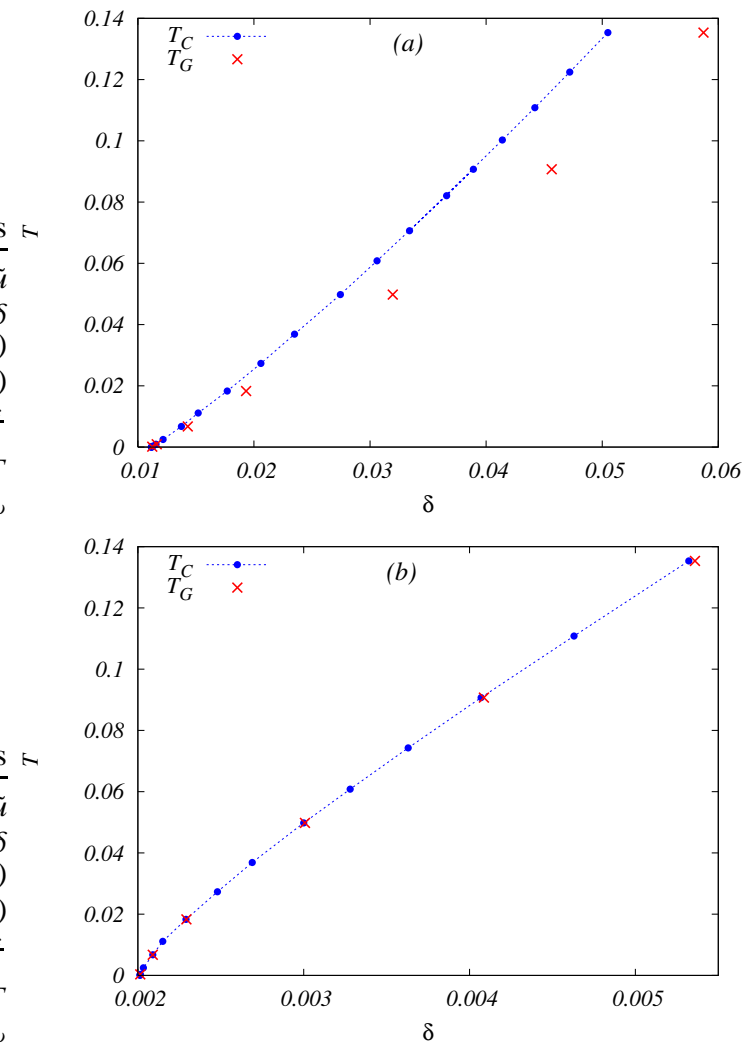

FIG. 4: (Color online) The transition lines $T_{c}(\delta)$ obtained for $z=3$, $d=2(a)$ and $z=3, d=3(b)$. The phase with broken symmetry is located below the line. The phase boundary $T_{c}(\delta)$ obeys $\left(\delta-\delta_{0}\right) \propto$ $T_{c} \log T_{c}$ for $d=2$ and $T_{c} \propto\left(\delta-\delta_{0}\right)^{3 / 4}$ for $d=3$ in agreement with the result by Millis. ${ }^{5}$ The crosses indicate the Ginzburg temperature $T_{G}$ for various choices of $\delta$.

14[15]19] for fixed $T$ and at different values of $\delta>\delta_{c}(T)$, observing the behavior of fixed-point values of the average order parameter $\phi_{0}$ (or, alternatively, the correlation length $\xi$ ) as $\delta$ approaches $\delta_{c}$. Typical results are plotted in Fig. 5 from which we read off the value of the exponent $\beta$ describing the decay of the order parameter upon approaching the transition line $\phi_{0} \propto\left(\delta-\delta_{c}\right)^{\beta}$. In the truly critical region (for $\delta-\delta_{c}$ small enough) one obtains $\beta \approx 0.11$ for $d=2$ and $\beta \approx 0.30$ for $d=3$. These results come out close to the correct classical values 0.125 and 0.31 , respectively. This is unlike the other critical exponents ( $\eta$ and the correlation length exponent $v$ ) which within our truncation differ by factors close to 2 from their correct values. Indeed, as discussed in Refs. 7,8, to obtain accurate values of the critical exponents in the Ising universality class, and in particular in $d=2$, one not only needs to consider the full partial differential equation governing the RG flow of the effective potential $U[\phi]$, but also the field dependence of the wave function renormalization and higher orders in the derivative expansion of the effective action $\frac{6}{6}$

From Fig. 5 we can extract the Ginzburg value $\delta_{G}$ below which true critical behavior is found at the chosen temperature $T$. Around $\delta_{G}$, the exponent $\beta$ exhibits a crossover from 

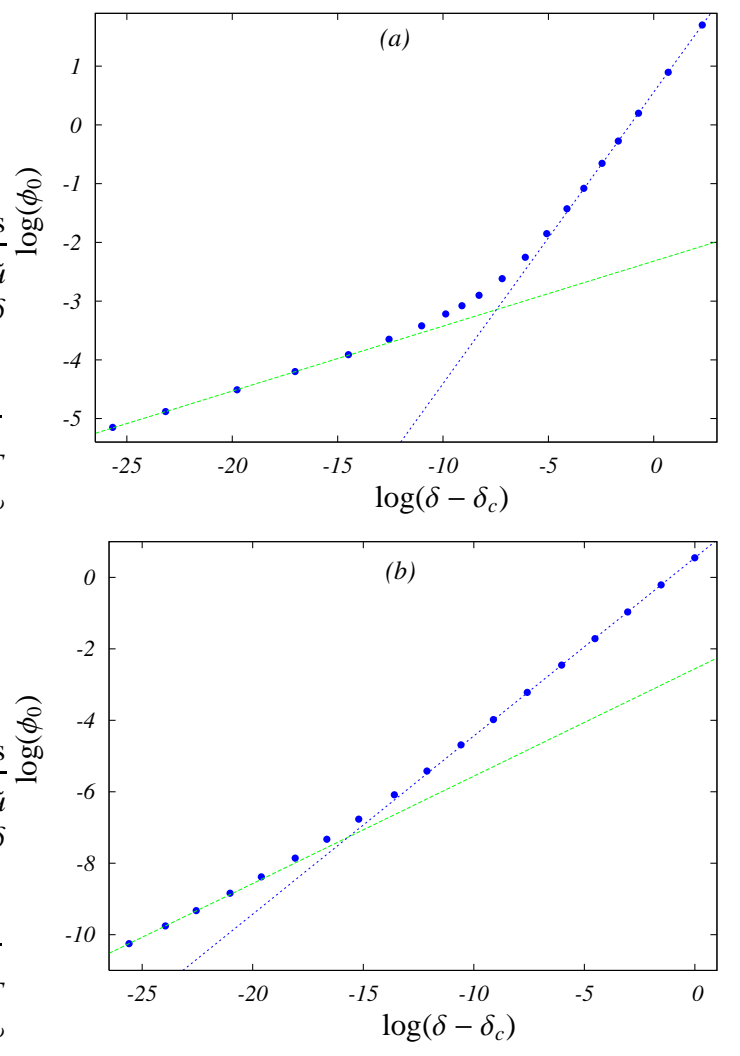

FIG. 5: (Color online) Order parameter $\phi_{0}$ as a function of $\left(\delta-\delta_{c}\right)$ at $T=e^{-5}$ for $z=3, d=2(a)$, and $z=3, d=3(b)$. The exponent $\beta$ governing the decay of $\phi_{0}$ upon approaching $\delta_{c}$ exhibits a crossover from a mean-field value $\beta=0.5$ to a non-gaussian $\beta \approx 0.11$ for $d=2$, and $\beta \approx 0.30$ for $d=3$. The intersection of the straight lines determines $\delta_{G}$.

its mean-field value $\beta=0.5$ to a non-gaussian value. In other words, $\delta_{G}$ marks the boundary of the non-gaussian critical region at a given $T$. At zero temperature, $\delta_{G}$ coincides with the quantum phase transition point $\delta_{0}$, since there the fluctuations are effectively $d+z>4$ dimensional, leading to mean-field behavior. Several Ginzburg points in the $\delta-T$ plane are plotted as $T_{G}(\delta)$ in Fig. 4, where they can be compared to the phase transition line. In three dimensions $T_{G}$ and $T_{c}$ almost coincide, such that $T_{G}$ provides an accurate estimate for $T_{c}$. In two dimensions a sizable region between $T_{G}$ and $T_{c}$ appears in the phase diagram. In that region, non-Gaussian classical fluctuations are present.

We stress that accounting for the anomalous exponent $\eta$ is necessary to describe the classical scaling regime, that is to obtain the plateaus in Figs. 2,3. Upon putting $\eta=0$ the scaling plateaus do not form. On the other hand, the shapes of the phase boundaries and the Ginzburg curves become very similar at $T \rightarrow 0$ in the present cases, where $d+z \geq 4$. Therefore our results for $T_{c}(\delta)$ are consistent with earlier studies,,$\frac{5}{,}$ where non-Gaussian critical fluctuations were not captured and the calculations were performed in the symmetric phase.

\section{B. $\mathbf{z}=2$}

In the case $z=2$ the flow equations (14]15[19) are significantly simplified, as all the integrals can be evaluated analytically. One obtains

$$
\begin{aligned}
\frac{d \tilde{\delta}}{d \log \Lambda} & =(\eta-2) \tilde{\delta}+2 \tilde{u}\left[\frac{2}{d} \frac{1}{(1+\tilde{\delta})^{2}}+\frac{4}{d} \tilde{T}^{-2} \Psi_{1}(y)\right] \\
& +3 \tilde{u} \tilde{\delta}\left[\frac{4}{d} \frac{1}{(1+\tilde{\delta})^{3}}-\frac{4}{d} \tilde{T}^{-3} \Psi_{2}(y)\right] \\
\frac{d \tilde{u}}{d \log \Lambda} & =(d-4+2 \eta) \tilde{u} \\
& +3 \tilde{u}^{2}\left[\frac{4}{d} \frac{1}{(1+\tilde{\delta})^{3}}-\frac{4}{d} \tilde{T}^{-3} \Psi_{2}(y)\right],
\end{aligned}
$$

and for the anomalous dimension,

$$
\begin{aligned}
\eta & =\frac{6}{d} \frac{\tilde{u} \tilde{\delta}}{(1+\tilde{\delta})^{5}}\left[2(1+\tilde{\delta})-\frac{8}{d+2}\right] \\
& +\frac{4 \tilde{u} \tilde{\delta}}{d} \tilde{T}^{-5}\left[\tilde{T} \Psi_{3}(y)+\frac{1}{d+2} \Psi_{4}(y)\right],
\end{aligned}
$$

where the argument of the polygamma functions is given by $y=1+(1+\tilde{\delta}) / \tilde{T}$.

The procedure to evaluate the phase diagram and the Ginzburg line is the same as in the previously discussed case $z=3$. In Fig. 6 we show results for the transition line $T_{c}(\delta)$ in two and three dimensions. We also show the Ginzburg temperature $T_{G}$ for various choices of $\delta$. In two dimensions, the transition line is consistent with the almost linear behavior $\left(\delta-\delta_{0}\right) \propto T_{c} \log \log T_{c} / \log T_{c}$, derived previously for the Ginzburg temperature in the symmetric phase $\stackrel{5}{\frac{5}{2}}$ However, a sizable region with non-Gaussian fluctuations opens between $T_{c}$ and $T_{G}$. In three dimensions, $T_{c}(\delta)$ obeys the expected 5 power law $T_{c}(\delta) \propto\left(\delta-\delta_{0}\right)^{\psi}$ with shift exponent $\psi=2 / 3$, and the Ginzburg temperature is very close to $T_{c}$ for any $\delta$.

\section{SUMMARY}

We have analyzed classical (thermal) and quantum fluctuations in the symmetry-broken phase near a quantum phase transition in an itinerant electron system. The analysis is restricted to the case of discrete symmetry breaking, where no Goldstone modes appear. Following Hertz ${ }^{4}$ and Millis,,$\frac{5}{-}$ we use an effective bosonic action for the order parameter fluctuations as a starting point. The renormalization of the Hertz action by fluctuations is obtained from a system of coupled flow equations, which are derived as an approximation to the exact flow equation for one-particle irreducible vertex functions in the functional RG framework. In addition to the renormalization of the effective mass and the four-point coupling, we also take the anomalous dimension $\eta$ of the order parameter fields into account. In the symmetry-broken phase, contributions to $\eta$ appear already at one-loop level. Quantum and thermal fluctuations are captured on equal footing. The flow equations are 

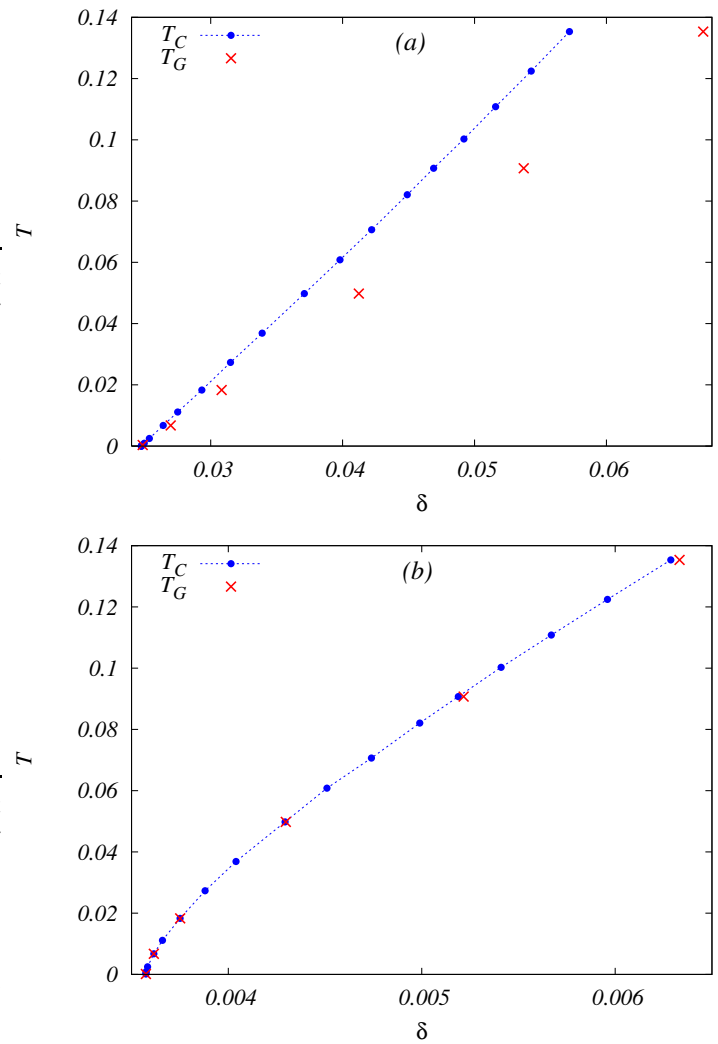

FIG. 6: (Color online) The transition lines $T_{c}(\delta)$ obtained for $z=2$, $d=2(a)$ and $z=2, d=3(b)$. The phase with broken symmetry is located below the line. For $d=2$ the phase boundary $T_{c}(\delta)$ is consistent with the relation $\left(\delta-\delta_{0}\right) \propto T_{c} \log \log T_{c} / \log T_{c}$ derived by Millis $\frac{5}{2}$ For $d=3$ it follows the expected power law $T_{c}(\delta) \propto$ $\left(\delta-\delta_{0}\right)^{2 / 3}$. The crosses indicate the Ginzburg temperature $T_{G}$ for various choices of $\delta$. applicable also in the immediate vicinity of the transition line at finite temperature, where fluctuations deviate strongly from Gaussian behavior.

We have computed the transition temperature $T_{c}$ as a function of the control parameter $\delta$ near the quantum critical point, approaching the transition line from the symmetry-broken phase. Explicit results were presented for dynamical exponents $z=2$ and $z=3$ in two and three dimensions. In all cases the functional form of $T_{c}(\delta)$ agrees with the behavior of the Ginzburg temperature above $T_{c}$ derived previously by Millis. $\frac{5}{-}$ We have also computed the Ginzburg temperature $T_{G}$ below $T_{c}$, above which non-Gaussian fluctuations become important. While $T_{G}$ and $T_{c}$ almost coincide in three dimensions, a sizable region between $T_{G}$ and $T_{c}$ opens in two dimensions.

It will be interesting to extend the present approach to the case of continuous symmetry breaking. In that case Goldstone modes suppress the transition temperature more strongly, and more extended regions governed by non-Gaussian fluctuations in the phase diagram appear.

\section{Acknowledgments}

We are grateful to B. Delamotte, J. Pawlowski, A. Rosch, and M. Salmhofer for valuable discussions, and to J. Bauer for a critical reading of the manuscript. We also like to thank M. Kircan for her pleasant collaboration at the initial stage of the project. PJ acknowledges support from the German Science Foundation through the research group FOR 723 and the Foundation for Polish Science through the START fellowship. PS acknowledges kind hospitality of G. Lonzarich and the Quantum Matter group at Cavendish Laboratory, University of Cambridge.
* Electronic address: p.jakubczyk@fkf.mpg.de

1 S. Sachdev, Quantum Phase Transitions (Cambridge University Press, Cambridge, U.K., 1999).

2 D. Belitz, T.R. Kirkpatrick, and T. Vojta, Rev. Mod. Phys. 70, 580 (2005).

${ }^{3}$ H. v. Löhneysen, A. Rosch, M. Vojta, and P. Wölfle, Rev. Mod. Phys. 79, 1015 (2007).

4 J.A. Hertz, Phys. Rev. B 14, 1165 (1976).

5 A.J. Millis, Phys. Rev. B 48, 7183 (1993).

${ }^{6}$ For a review of the one-particle irreducible version of the exact RG with a broad range of applications, see, J. Berges, N. Tetradis, and C. Wetterich, Phys. Rep. 363, 223 (2002).

7 H. Ballhausen, J. Berges, and C. Wetterich, Phys. Lett. B 582, 144 (2004).

${ }^{8}$ L. Canet, B. Delamotte, D. Mouhanna, and J. Vidal, Phys. Rev. B 68, 064421 (2003).

9 A. Sinner, N. Hasselmann, and P. Kopietz, J. Phys.: Condens. Matter 20, 075208 (2008).

10 C. Wetterich, Phys. Rev. B 77, 064504 (2008).

11 W. Metzner, D. Rohe, and S. Andergassen, Phys. Rev. Lett. 91, 066402 (2003).
12 L. Dell' Anna and W. Metzner, Phys. Rev. B 73, 045127 (2006).

13 A. Rosch and P. Wölfle, J. Low Temp. Phys. 147, 165 (2007).

14 A. Abanov and A.V. Chubukov, Phys. Rev. Lett. 93, 255702 (2004).

15 A. Neumayr and W. Metzner, Phys. Rev. B 58, 15449 (1998).

16 C. Kopper and J. Magnen, Ann. Henri Poincaré 2, 513 (2001).

17 A.V. Chubukov and D.L. Maslov, Phys. Rev. B 68, 155113 (2003), and references therein.

18 J. Rech, C. Pepin, and A.V. Chubukov, Phys. Rev. B 74, 195126 (2006).

19 N. Nagaosa, Quantum Field Theory in Strongly Correlated Electronic Systems (Springer, Berlin, Heidelberg, Germany, 1999).

20 A. Rosch, Phys. Rev. B 64, 174407 (2001).

21 C. Wetterich, Phys. Lett. B 301, 90 (1993).

22 B. Delamotte, D. Mouhanna and M. Tissier, Phys.Rev. B 69 134413 (2004); B. Delamotte, arXiv:cond-mat/0702365 (2007).

23 W. Metzner, Prog. Theor. Phys. Suppl. 160, 58 (2005).

${ }^{24}$ H. Gies, arXiv:hep-ph/0611146 (2006).

25 D.F. Litim, Phys. Rev. D 64, 105007 (2001).

26 N. Goldenfeld, Lectures on Phase Transitions and the Renormalizaion Group (Perseus Publishing, 1992). 
27 Note that the shape of the Ginzburg curve drawn in Fig. 3 of Ref. 5 does not correspond to the formula for $T_{G}(\delta)$ derived in the same paper.
28 J. Braun and H. Gies, JHEP 06, 024 (2006). 